Published in final edited form as:

Oral Oncol. 2018 May ; 80: 9-15. doi:10.1016/j.oraloncology.2018.02.021.

\title{
The risk of carotid stenosis in head and neck cancer patients after radiation therapy
}

\author{
David J. Carpenter ${ }^{\mathrm{a}}$, Yvonne M. Mowery ${ }^{\mathrm{b}}$, Gloria Broadwater ${ }^{\mathrm{c}}$, Anna Rodrigues ${ }^{\mathrm{b}}$, Amy J. \\ Wisdom $^{a}$, Jennifer A. Dorth ${ }^{\mathrm{e}}$, Pretesh R. Patel ${ }^{f}$, Cynthia K. Shortell ${ }^{d}$, Robert Clough ${ }^{b}$, David \\ M. Brizel ${ }^{\text {b,d }}$ \\ ${ }^{\text {aD }}$ uke University School of Medicine, Durham, NC, USA \\ bDepartment of Radiation Oncology, Duke University Health Syste, USA \\ 'Department of Biostatistics, Duke University Health System, USA \\ dDepartment of Surgery, Duke University Health System, USA \\ eDepartment of Radiation Oncology, Case Western Reserve University, Cleveland, OH, USA \\ fDepartment of Radiation Oncology, Winship Cancer Institute of Emory University, Atlanta, GA, \\ USA
}

\section{Abstract}

Objectives: Head and neck radiotherapy (RT) is a risk factor for cerebrovascular disease. We performed a retrospective cohort study to evaluate carotid artery stenosis (CAS) incidence in head and neck cancer (HNC) patients undergoing RT, characterizing associated risk factors.

Materials and methods: Records were retrospectively reviewed for HNC patients undergoing carotid ultrasound screening after definitive or adjuvant RT between January 2000 and May 2016. CAS was defined as $\mathbf{2 5 0 \%}$ stenosis on imaging, stroke, or transient ischemic attack. Actuarial CAS rates were calculated by Kaplan-Meier method. Univariate and multivariate analyses predicted CAS risk based on carotid dosimetric and clinical parameters.

Results: 366 patients met inclusion criteria. Median time from RT completion to last follow-up was 4.1 yr. Actuarial risk for CAS was 29\% (95\% CI 22-36\%) at 8years. Univariate analysis showed that smoking (HR 1.7; 95\% CI 1.1-2.7), hyperlipidemia (HR 1.6; 95\% CI 1.03-2.6), diabetes (HR 2.8; 95\% CI 1.6-4.8), coronary artery disease (HR 2.4; 95\% CI 1.4-4.2), and peripheral artery disease (HR 3.6; 95\% CI 1.1-11.6) were significantly associated with increased CAS. In multivariate analysis, diabetes was predictive of time to CAS (HR 1.9; 95\% CI 1.1-3.4). Carotid dose parameters were not significantly associated with CAS.

\footnotetext{
*Corresponding author at: Duke University Health System, Box 3085, Durham, NC 27710, USA. david.brizel@ duke.edu (D.J. Carpenter).

Conflict of interest

None declared.

Appendix A. Supplementary material

Supplementary data associated with this article can be found, in the online version, at https://doi.org/10.1016/j.oraloncology. 2018.02.021
} 
Conclusions: CAS incidence is high after head and neck radiotherapy, gradually rising over time. No clear dose-response effect between carotid dose and CAS was identified for HNC patients. Carotid artery screening and preventative strategies should be employed in this high-risk patient population.

\section{Keywords}

Carotid stenosis; Head and neck neoplasms; Radiotherapy; Radiation effects; Cerebrovascular disorders

\section{Introduction}

The role of post-treatment screening for asymptomatic carotid artery stenosis (CAS) in the head and neck cancer (HNC) population is unclear. The United States Preventive Task Force does not recommend CAS screening in the general population due to low CAS prevalence $(<5 \%)$ [1]. Addressing higher risk populations, guidelines from the Society of Vascular Surgeons and the American Society of Neuroimaging determined CAS screening to be costeffective for reducing stroke when CAS prevalence is $220 \%$, and potentially cost-effective when CAS prevalence is between 5\% and 20\% [2,3]. Reports supporting these guidelines differ in their definitions of CAS, ranging from $50 \%$ to $80 \%$ luminal reduction [4,5]. In those defining CAS as $250 \%$ luminal reduction, independent predictors of high CAS prevalence (9-21\%) include age, diabetes mellitus (DM), coronary artery disease (CAD), smoking, and history of stroke or transient ischemic attack (TIA) [5-9]. Current CAS screening recommendations do not include patients undergoing radiation therapy (RT) for HNC.

Head and neck RT is an independent risk factor for stroke and asymptomatic CAS. RT correlated with high rates of stroke in two retrospective series comparing HNC patients to matched controls from population-based stroke registries (relative risk 2.1-10.1) [10,11]. Several Surveillance, Epidemiology, and End Results analyses of HNC patients showed that, compared to surgery alone, RT use was associated with an increased 10-year risk of stroke [12,13], and 15-year risk of fatal stroke [14]. RT also correlated with high rates of asymptomatic stenosis in multiple cross-sectional studies of HNC patients, with CAS prevalence ranging from $11.7 \%$ at a mean of 72 months post-RT to $19.8 \%$ at a mean of 24months post-RT [15-19].

CAS screening post-RT for HNC began at our institution in 2000. Doppler ultrasound (US) is typically performed $12-18$ months following RT completion with subsequent screening every 3years where CAS is $<50 \%$. Patients with $250 \%$ CAS are screened annually and referred to vascular surgery for further evaluation and management. Our initial report of 224 asymptomatic HNC patients revealed a 14\% actuarial rate of CAS at 4years post-RT [19]. The current study assesses asymptomatic CAS and symptomatic cerebrovascular disease in an expanded cohort with longer follow-up. 


\section{Methods}

\section{Subject selection}

Records were retrospectively reviewed under an institutional review board-approved protocol for all adult patients who underwent carotid screening following definitive curativeintent or post-operative RT for non-metastatic HNC at Duke University Medical Center between January 2000 and May 2016. Exclusion criteria included previous CAS, stroke, or RT to the neck, or failure to obtain baseline carotid screening within 2years after RT completion (Fig. 1).

Patient demographics, disease stage, primary disease site, histology, smoking status, hypertension (HTN), hyperlipidemia (HLD), DM, CAD, peripheral artery disease (PAD), congestive heart disease, atrial fibrillation, receipt of chemotherapy, neck dissection status, and occurrence of stroke or TIA were obtained by chart review. The following RT parameters were recorded: technique (intensity modulated radiation therapy [IMRT], 2D, or 3D conformal radiation therapy), total dose, schedule (conventional [1.8-2.0Gy/fraction, 5treatments/week], accelerated [1.8-2.0Gy/fraction, $>5$ treatments/week], accelerated hyperfractionation [ $<1.25 \mathrm{~Gy} /$ fraction, 10 treatments/week], or hypofractionation [ $>2 \mathrm{~Gy} /$ fraction once daily]), and sidedness (bilateral or unilateral). Baseline carotid artery imaging (within 2years of RT completion) and follow-up carotid artery imaging results were reviewed. CAS screening practice evolved empirically from its inception in 2000. By 2005, most patients underwent post-treatment screening by doppler US within 1-2years post-RT followed by surveillance carotid US approximately every 3years or more frequently if indicated by abnormal imaging results or symptoms. Patients with clinically significant or worsening CAS were referred to vascular surgery for further evaluation and management.

\section{Carotid artery stenosis and dosimetric parameters}

CAS was analyzed as a composite outcome including both asymptomatic carotid stenosis and cerebrovascular events (CVEs). Asymptomatic carotid stenosis was defined as $\mathbf{2 5 0 \%}$ reduction in luminal diameter of common and/or internal carotid artery on carotid artery imaging (ultrasound, arteriogram, computed tomography angiogram, and/or magnetic resonance angiogram). CVEs were defined as stroke and/or TIAs. Patients not undergoing US screening within 2years post-RT due to CVEs during that timeframe $(n=6)$ were excluded due to the high likelihood of pre-existing asymptomatic CAS. Carotid arteries (extending from clavicle to entry into temporal bone) and carotid bulbs ( $2 \mathrm{~cm}$ above and below carotid bifurcation) were contoured on the radiation treatment planning computed tomography (CT) scan. The following dosimetric parameters were calculated for each carotid artery and bulb: maximum dose, mean dose, and partial organ volumes receiving 40, 50, 60, or 70Gy (V40, V50, etc.). Maximum and mean carotid doses were analyzed in 10Gy increments.

\section{Statistical analysis}

Actuarial rates of composite CVEs and asymptomatic CAS following RT completion were calculated by Kaplan-Meier product limit method. The time interval was calculated from the completion of RT until development of carotid artery stenosis detected on imaging. Patients 
were censored for composite CAS at death or last follow-up appointment. Asymptomatic CAS was censored at CVE onset or date of last carotid imaging. Univariate Cox proportional hazards regression analyses were used to predict time to composite CAS development using the following factors: age, sex, smoking, HTN, HLD, DM, CAD, PAD, neck dissection, receipt of chemotherapy, and RT sidedness. Univariate analysis per patient included total RT dose. Analysis per individual carotid artery included maximum dose, mean dose, V40, V50, V60, and V70 values for the entire carotid and the carotid bulb. Multivariate Cox proportional hazards models were constructed per patient. Variables were retained in the model based on the following criteria: univariate p-value (1) $<0.05$ or $(2)<0.07$ for variables significantly associated with carotid artery stenosis in previous reports. Statistical analyses were conducted using SAS v 9.4 (SAS Institute, Inc., Cary, NC), and actuarial plots were created using Spotfire S+v. 8.1 (TIBCO, Palo Alto, CA).

\section{Results}

A total of 1295 patients underwent RT for non-metastatic HNC between January 2000 and May 2016. Three hundred sixty-one patients did not undergo baseline carotid US screening within two years due to HNC recurrence, death, second malignancy, severe comorbid illness, or CVE. Additionally, 458 patients did not undergo baseline carotid screening within two years of RT completion for other reasons while 110 patients underwent baseline screening $>24$ months post-RT completion without a documented reason for delayed screening. The remaining 366 patients met inclusion criteria (Fig. 1A). As our institutional screening practice evolved over time, an increasing proportion of patients completed baseline screening within 2years: 3\% (4/156) from 2000 to $01,31 \%$ (64/206) from 2007 to 08 , and $40 \%$ (77/193) from 2013 to 2014.

Table 1 shows baseline patient characteristics. Patients were predominantly male (80\%) with a mean age of 59.6years (range: 23-97). Squamous cell carcinoma comprised 95\% of cases with oropharynx being the most common primary tumor site (63\%). Most patients had locally advanced disease ( $16 \%$ stage III; $71 \%$ stage IV). Median time from RT completion to last follow-up was 4.1years (interquartile range, 2.3-6.8).

Table 2 summarizes treatment characteristics, carotid imaging studies, and outcomes (asymptomatic CAS, stroke, TIA). IMRT was used for $91 \%$ of patients, and RT was administered to bilateral neck in $77 \%$ of cases. Most patients underwent conventionally fractionated radiation therapy (72\%) and received concurrent chemotherapy (75\%). Neck dissection was performed in $30 \%$ of cases. Patients underwent an average of two carotid imaging studies (range, 1-8) after RT. Carotid ultrasound was the predominant imaging modality $(95 \%)$.

Stroke occurred in 18 patients (5\%), and TIA in 13 patients (4\%). Asymptomatic CAS was documented prior to 6 of $18 \mathrm{~S}$. CAS management beforehand for these patients included carotid stenting $(n=1)$, medical management $(n=2)$, or observation alone $(n=3)$.

Asymptomatic CAS was documented prior to 7 of 13 TIAs. These patients underwent carotid stenting $(n=1)$, carotid endarterectomy $(n=1)$, medical management $(n=3)$, or observation alone $(\mathrm{n}=2)$ after CAS diagnosis on imaging. 
Asymptomatic CAS was observed in 58 patients (16\%). Fourteen of 58 patients (24\%) with asymptomatic CAS were prescribed a new or higher aspirin dose or other anti-coagulant medication, 13 patients $(22 \%)$ were prescribed a new anti-hypertensive or lipid-lowering medication, 7 patients (12\%) underwent carotid stent placement, and 2 patients (3\%) underwent carotid endarterectomy.

Fig. 2 shows the actuarial incidence of composite and asymptomatic CAS. Cumulative composite CAS incidence (Fig. 2A) was $11 \%$ at 2 years, $20 \%$ at 5years, and $29 \%$ at 8 years while asymptomatic CAS incidence (Fig. 2B) was $12 \%$ at 2years, $23 \%$ at 5years, and $34 \%$ at 8years.

Dosimetric parameters for carotid arteries and carotid bulbs were calculated for patients $(\mathrm{n}=308)$ with available CT simulation imaging (Supplemental Table S1). Mean carotid artery dose was 48 $\pm 19 \mathrm{~Gy}$. On univariate analysis per patient (Table 3A), age, smoking, DM, and CAD were significantly associated with composite CAS. Multivariate analysis per patient (Table 3B) demonstrated that DM was predictive of composite CAS (HR 1.9, 95\% CI 1.13.4; $p=0.03$ ) when controlling for age, sex, smoking, HLD and CAD.

Univariate analysis of composite CAS per carotid artery (Table 4) demonstrated significant association with smoking, HLD, DM, CAD, and PAD. No carotid artery or carotid bulb dosimetric parameters correlated significantly with composite CAS on univariate analysis. Receiver operating characteristic (ROC) curve analysis for dosimetric parameters showed no cutpoints associated with CAS. Multivariate analysis was not performed for composite CAS per carotid artery because composite CAS did not correlate with carotid-specific dosimetric parameters on univariate analysis.

\section{Discussion}

Since 2000, we gradually adopted carotid US screening of patients after RT for HNC because of the correlation between this modality and an increased risk for CVEs [10-12,15-18,20-29]. The current report represents the largest study of the prevelance of asymptomatic CAS after RT for HNC. The American Society of Neuroimaging and Society for Vascular Surgery cite cutoffs for carotid luminal diameter reduction ranging from 50\% (5 of 9 reports) to $80 \%$ [4-9,30-32]. Accordingly, we adopted a conservative definition of $250 \%$ luminal reduction. Our previous analysis demonstrated a nonsignificant trend toward an increased risk of CAS with an increasing mean dose to the carotid bulb (1.4 HR for CAS for every 10Gy, 95\% CI 0.8-2.4) [19]. The present expanded cohort with longer follow-up demonstrates no correlation between CAS and carotid artery or bulb dosimetric parameters for HNC patients.

The cost effectiveness of CAS screening depends on CAS prevalence. The Society for Vascular Surgery guidelines indicate that "the prevalence of significant carotid artery stenosis may be high enough, depending on the time between radiotherapy exposure and screening, to justify routine carotid screening" in this patient population [2]. The American Society of Neuroimaging, while not addressing populations receiving head and neck RT, determined that CAS screening is cost-effective for reducing stroke where CAS prevalence 
is $\geq 20 \%$, and some studies suggest that it is cost-effective for populations with 5-20\% prevalence $[2,3,33]$. In this context, the current data suggest that it would be reasonable to start screening HNC patients for asymptomatic CAS between 2 and 5years following RT completion (asymptomatic CAS incidence $12-23 \%$ in this series).

No dose-response effect was observed between carotid artery dose and CAS. The typical carotid doses >40Gy among HNC patients probably exceed the threshold for CAS development, missing the dose range in which a dose-response effect could be observed. Lower carotid artery doses were present for contralateral arteries outside unilateral treatment fields, with carotid bulb maximum doses $<40 \mathrm{~Gy}$ for 78 of 616 arteries. Within this lower range of carotid artery dose, ROC analysis was likely underpowered for detecting dosimetric cutpoints associated with CAS. Lymphoma survivors receiving head and neck RT present a comparison of lower RT doses (typically 40Gy maximum), usually in the absence of competing CAS risk factors. Children's Oncology Group (COG) guidelines recommend CAS screening by carotid ultrasound starting at ten years after RT completion for lymphoma survivors receiving head and neck RT $\geq 40 \mathrm{~Gy}$, while annual neurologic examinations are recommended for doses $\geq 18 \mathrm{~Gy}$ [34]. COG guidelines for CAS screening rely primarily on significant correlations of RT dose with non-CAS vascular pathologies [35-37]. The only guideline-supporting report of CAS screening in lymphoma patients that analyzed dose dependency demonstrated a non-significant trend towards higher median low-cervical radiation dose for those who developed carotid artery disease (38Gy; range, 30-57Gy) compared to those who did not (36Gy; range, 13-76Gy) [37]. A similar report examining 40 HNC patients who received $255 \mathrm{~Gy}$ cervical radiation found no difference in RT dose between those who did and did not develop significant CAS [27]. In both cases, the lack of statistically significant dose dependence was attributed to narrow dose ranges in context of the widely-held belief that RT toxicity is dose-dependent.

Time from RT completion may be an additional confounding factor of dose dependency of CAS in HNC survivors [38]. The median follow-up of 4 years in the current study may be too short to detect a statistically significant dose response effect. It may also underestimate the long term risk for development of this condition. Longer follow-up times were limited in the present study by HNC progression, non-HNC death, and lack of carotid screening beyond a single doppler US within 2 years in $>50 \%$ of the studied population. Previous reports correlating CVEs with prior RT included follow-up periods exceeding 10 years [12-14,39]. Cheng et al. discovered that patients who had neck irradiation >5years prior have an eightfold higher risk of CAS compared to those with a post-RT time interval <5years $(p<0.001)$ [15]. In summary, whether neck doses above a threshold for CAS development or follow-up duration influenced the observed lack of dose dependency in the current study, HNC patients with multiple non-RT arteriosclerotic risk factors demonstrated high CAS incidence and warrant post-RT screening. The high incidence of CAS in this series and the increasing incidence of HPV-related OPC where many of these risk factors are absent highlights the need to investigate dose de-escalation strategies in these patients.

Characterizing RT for $\mathrm{HNC}$ as an isolated CAS risk factor is increasingly important as HNC patients shift towards a younger population with Human Papillomavirus (HPV)-driven disease, limited prior tobacco use, and improved prognosis. Younger patients have a longer 
lifetime risk of ischemic stroke following RT. For example, a study of 10,172 HNC patients demonstrated that treatment with RT and/or chemotherapy conferred a 1.8-fold higher risk for stroke among patients younger than 55years while no significant difference in stroke incidence was observed for patients aged 255 years [40]. A similar report compared cases of ischemic stroke in patients hospitalized for nasopharyngeal carcinoma (NPC) versus appendectomy (control group). Lee et al. found that NPC patients aged 35-54 had a 1.7-fold higher risk of ischemic stroke compared to the control group after adjusting for other factors [41]. In contrast, ischemic stroke risk did not differ significantly between NPC and appendectomy patients aged 55-64years $(\mathrm{HR}=0.87$; 95\% CI, 0.56-1.33; $p=0.524)$.

The current study has several limitations. The $23 \%$ of patients who underwent CAS screening may not accurately represent the general HNC population. Because the majority of post-RT strokes and TIAs were managed at outside medical centers, confirming the presence of large vessel disease was often impossible. The composite CAS endpoint likely overestimates outcomes related to carotid artery stenosis by including all post-RT strokes and TIAs. While approximately half of strokes in the general population are estimated to be of large-artery origin [42], the distribution and pathology of vascular disease leading to strokes among patients after cervical irradiation may differ from other patients. RT technique and fractionation schedules were not included in the multivariate dosimetric analysis, as these factors were likely inconsequential in the absence of any trends suggesting a dose-response effect.

\section{Conclusion}

The high incidence of asymptomatic CAS warrants carotid artery screening after RT in the HNC population despite the absence of a discernible dose-response relationship.

\section{Supplementary Material}

Refer to Web version on PubMed Central for supplementary material.

\section{Acknowledgements}

This research was supported by the National Institutes of Health (NIH) under award number UL1TR001117. The content is solely the responsibility of the authors and does not necessarily represent the official view of the NIH.

\section{References}

[1]. Wolff T, Guirguis-Blake J, Miller T, Gillespie M, Harris R, Screening for carotid artery stenosis: an update of the evidence for the US Preventive Services Task Force, Ann Intern Med 147 (12) (2007) 860-870. [PubMed: 18087057]

[2]. Ricotta JJ, AbuRahma A, Ascher E, Eskandari M, Faries P, Lal BK, Updated Society for Vascular Surgery guidelines for management of extracranial carotid disease, J Vase Surg 54 (3) (2011) e1e31.

[3]. Qureshi AI, Alexandrov AV, Tegeler CH, Hobson RW 2nd, Dennis Baker J, Hopkins LN, Guidelines for screening of extracranial carotid artery disease: a statement for healthcare professionals from the multidisciplinary practice guidelines committee of the American Society of Neuroimaging; cosponsored by the Society of Vascular and Interventional Neurology, J Neuroimaging 17 (1) (2007) 19-47. [PubMed: 17238868] 
[4]. Durand DJ, Perler BA, Roseborough GS, et al., Mandatory versus selective preoperative carotid screening: a retrospective analysis, Ann Thorac Surg 78 (1) (2004) 159-166. [PubMed: 15223422]

[5]. D'Agostino RS, Svensson LG, Neumann DJ, Balkhy HH, Williamson WA, Shahian DM, Screening carotid ultrasonography and risk factors for stroke in coronary artery surgery patients, Ann Thorac Surg 62 (6) (1996) 1714-1723. [PubMed: 8957376]

[6]. Fukuda I, Gomi S, Watanabe K, Seita J, Carotid and aortic screening for coronary artery bypass grafting, Ann Thorac Surg 70 (6) (2000) 2034-2039. [PubMed: 11156116]

[7]. Fukuda I, Ohuchi H, Sato M, Sato F, Wada M, Carotid screening with duplex scanning before coronary artery bypass, Jpn J Thorac Cardiovasc Surg 44 (4) (1996) 478-483.

[8]. Rath PC, Agarwala MK, Dhar PK, et al., Carotid artery involvement in patients of atherosclerotic coronary artery disease undergoing coronary artery bypass grafting, Indian Heart J 53 (6) (2001) 761-765. [PubMed: 11838931]

[9]. Tanimoto S, Ikari Y, Tanabe K, Yachi S, Nakajima H, Nakayama T, et al., Prevalence of carotid artery stenosis in patients with coronary artery disease in Japanese population, Stroke 36 (10) (2005) 2094-2098. [PubMed: 16179563]

[10]. Dorresteijn LD, Kappelle AC, Boogerd W, et al., Increased risk of ischemic stroke after radiotherapy on the neck in patients younger than 60 years, J Clin Oncol 20 (1) (2002) 282-288. [PubMed: 11773180]

[11]. Haynes JC, Machtay M, Weber RS, Weinstein GS, Chalian AA, Rosenthal DI, Relative risk of stroke in head and neck carcinoma patients treated with external cervical irradiation, Laryngoscope 112 (10) (2002) 1883-1887. [PubMed: 12368635]

[12]. Smith GL, Smith BD, Buchholz TA, et al., Cerebrovascular disease risk in older head and neck cancer patients after radiotherapy, J Clin Oncol 26 (31) (2008) 5119-5125. [PubMed: 18725647]

[13]. Hong JC, Kruser TJ, Gondi V, et al., Risk of cerebrovascular events in elderly patients after radiation therapy versus surgery for early-stage glottic cancer, Int J Radiat Oncol Biol Phys 87 (2) (2013) 290-296. [PubMed: 23906930]

[14]. Swisher-McClure S, Mitra N, Lin A, et al., Risk of fatal cerebrovascular accidents after external beam radiation therapy for early-stage glottic laryngeal cancer, Head Neck 36 (5) (2014) 611616. [PubMed: 23595858]

[15]. Cheng SW, Wu LL, Ting AC, Lau H, Lam LK, Wei WI, Irradiation-induced extracranial carotid stenosis in patients with head and neck malignancies, Am J Surg 178 (4) (1999) 323-328. [PubMed: 10587192]

[16]. Chang Y-J, Chang T-C, Lee T-H, Ryu S-J, Predictors of carotid artery stenosis after radiotherapy for head and neck cancers, J Vasc Surg 50 (2) (2009) 280-285. [PubMed: 19631860]

[17]. Carmody BJ, Arora S, Avena R, et al., Accelerated carotid artery disease after high-dose head and neck radiotherapy: is there a role for routine carotid duplex surveillance?, J Vasc Surg 30 (6) (1999) 1045-1051. [PubMed: 10587388]

[18]. Lam WW, Leung SF, So NM, et al., Incidence of carotid stenosis in nasopharyngeal carcinoma patients after radiotherapy, Cancer 92 (9) (2001) 2357-2363. [PubMed: 11745291]

[19]. Dorth JA, Patel PR, Broadwater G, Brizel DM, Incidence and risk factors of significant carotid artery stenosis in asymptomatic survivors of head and neck cancer after radiotherapy, Head Neck 36 (2014) 215-219. [PubMed: 23554082]

[20]. Silverberg GD, Britt RH, Goffinet DR, Radiation-induced carotid artery disease, Cancer 41 (1) (1978) 130-137. [PubMed: 626923]

[21]. Moritz MW, Higgins RF, Jacobs JR, Duplex imaging and incidence of carotid radiation injury after high-dose radiotherapy for tumors of the head and neck, Arch Surg 125 (9) (1990) 11811183. [PubMed: 2205173]

[22]. Dubec J, Munk P, Tsang V, et al., Carotid artery stenosis in patients who have undergone radiation therapy for head and neck malignancy, Br J Radiol 71 (848) (1998) 872-875. [PubMed: 9828801]

[23]. Friedlander AH, Eichstaedt RM, Friedlander IK, Lambert PM, Detection of radiation-induced, accelerated atherosclerosis in patients with osteoradionecrosis by panoramic radiography, J Oral Maxillofac Surg 56 (4) (1998) 455-459. [PubMed: 9541345] 
[24]. Freymiller E, Sung E, Friedlander A, Detection of radiation-induced cervical atheromas by panoramic radiography, Oral Oncol 36 (2) (2000) 175-179. [PubMed: 10745169]

[25]. Halak M, Fajer S, Ben-Meir H, Loberman Z, Weller B, Karmeli R, Neck irradiation: a risk factor for occlusive carotid artery disease, Eur J Vasc Endovasc Surg 23 (4) (2002) 299-302. [PubMed: 11991689]

[26]. Brown PD, Foote RL, McLaughlin MP, et al., A historical prospective cohort study of carotid artery stenosis after radiotherapy for head and neck malignancies, Int J Radiat Oncol Biol Phys 63 (5) (2005) 1361-1367. [PubMed: 16169673]

[27]. Steele SR, Martin MJ, Mullenix PS, Crawford JV, Cuadrado DS, Andersen CA, Focused highrisk population screening for carotid arterial stenosis after radiation therapy for head and neck cancer, Am J Surg 187 (5) (2004) 594-598. [PubMed: 15135672]

[28]. Martin JD, Buckley AR, Graeb D, Walman B, Salvian A, Hay JH, Carotid artery stenosis in asymptomatic patients who have received unilateral head-and-neck irradiation, Int J Radiat Oncol Biol Phys 63 (4) (2005) 1197-1205. [PubMed: 15978738]

[29]. Gujral DM, Chahal N, Senior R, Harrington KJ, Nutting CM, Radiation-induced carotid artery atherosclerosis, Radiother Oncol 110 (1) (2014) 31-38. [PubMed: 24044796]

[30]. Hill AB, Obrand D, Steinmetz OK, The utility of selective screening for carotid stenosis in cardiac surgery patients, J Cardiovasc Surg 40 (6) (1999) 829-836. [PubMed: 10776713]

[31]. Ascher E, DePippo P, Salles-Cunha S, Marchese J, Yorkovich W, Carotid screening with duplex ultrasound in elderly asymptomatic patients referred to a vascular surgeon: is it worthwhile?, Ann Vasc Surg 13 (2) (1999) 164-168. [PubMed: 10072455]

[32]. Ascher E, Hingorani A, Yorkovich W, Ramsey PJ, Salles-Cunha S, Routine preoperative carotid duplex scanning in patients undergoing open heart surgery: is it worthwhile?, Ann Vasc Surg 15 (6) (2001) 669-678. [PubMed: 11769149]

[33]. Yin D, Carpenter JP, Cost-effectiveness of screening for asymptomatic carotid stenosis, J Vasc Surg 27 (2) (1998) 245-255. [PubMed: 9510279]

[34]. Bhatia S, Casillas J, Hudson MM, et al., Long-term follow-up guidelines for survivors of childhood, adolescent, and young adult cancers, Children's Oncology Group, Arcadia (CA), 2008.

[35]. Martin R, Wiernik P, Dose and treatment factors in radiation-related pericardial effusion associated with the mantle technique for Hodgkin's disease, Cancer 35 (1975).

[36]. Morton DL, Glancy DL, Joseph WL, Adkins PC, Management of patients with radiation-induced pericarditis with effusion: a note on the development of aortic regurgitation in two of them, Chest 64 (3) (1973) 291-297. [PubMed: 4127171]

[37]. Hull MC, Morris CG, Pepine CJ, Mendenhall NP, Valvular dysfunction and carotid, subclavian, and coronary artery disease in survivors of Hodgkin lymphoma treated with radiation therapy, $\mathrm{J}$ Am Med Assoc 290 (21) (2003) 2831-2837.

[38]. Fajardo L, Berthrong M, Vascular lesions following radiation, Pathol Annu 23 (1988) 297-330. [PubMed: 3387138]

[39]. Dorresteijn LD, Kappelle AC, Scholz NM, et al., Increased carotid wall thickening after radiotherapy on the neck, Eur J Cancer 41 (7) (2005) 1026-1030. [PubMed: 15862751]

[40]. Huang YS, Lee CC, Chang TS, et al., Increased risk of stroke in young head and neck cancer patients treated with radiotherapy or chemotherapy, Oral Oncol 47 (11) (2011) 1092-1097. [PubMed: 21852184]

[41]. Lee CC, Su YC, Ho HC, et al., Increased risk of ischemic stroke in young nasopharyngeal carcinoma patients, Int J Radiat Oncol Biol Phys 81 (5) (2011) e833-e838. [PubMed: 21570205]

[42]. Inzitari D, Eliasziw M, Gates P, et al., The causes and risk of stroke in patients with asymptomatic internal-carotid-artery stenosis, N Engl J Med 342 (23) (2000) 1693-1701. [PubMed: 10841871] 


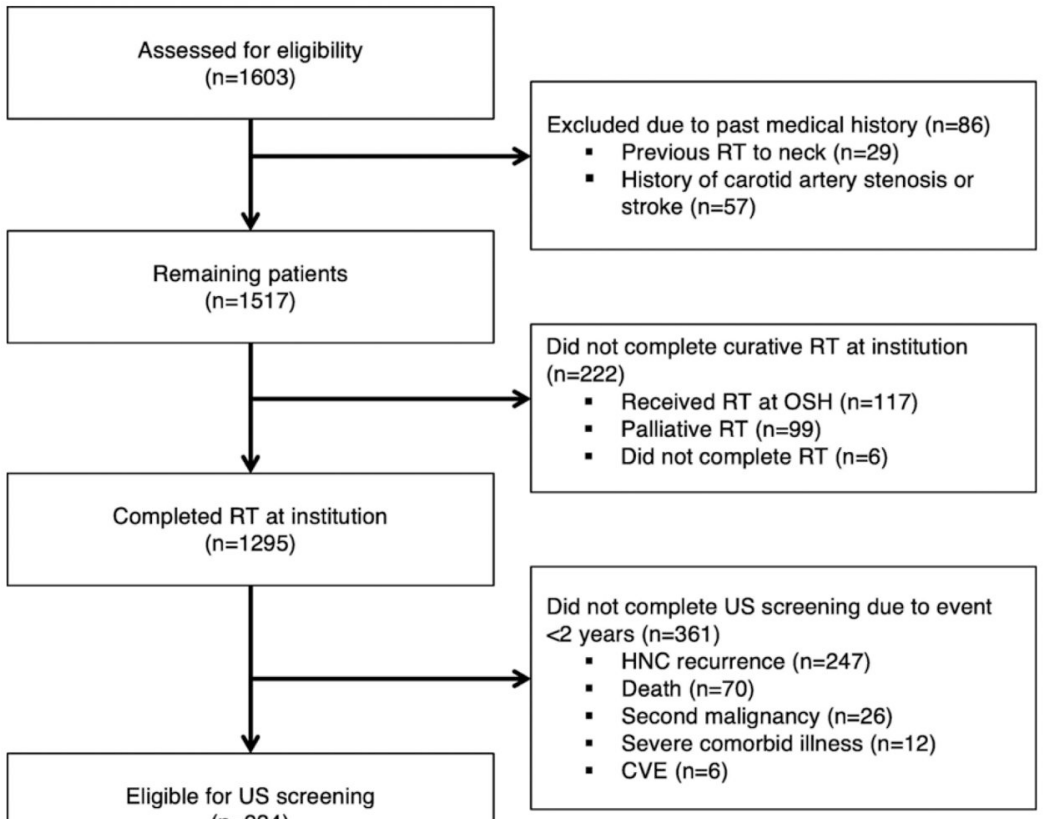
$(n=934)$

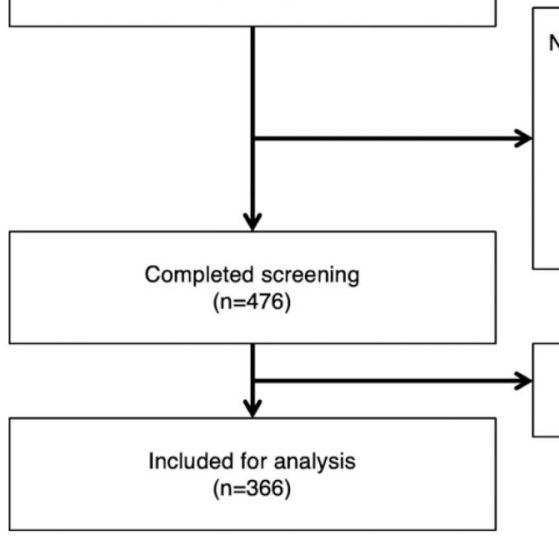

Not screened $(n=458)$

- Follow-up at OSH $(n=69)$

- Follow-up with ENT only ( $n=58)$

- Follow-up before initiation of practice $(n=216)$

- Lost to follow-up ( $n=54)$

- Scan ordered, not completed $(n=12)$

- Still within 2-year window $(n=49)$

Baseline carotid US >2 years from RT completion $(n=110)$

Fig. 1.

Consort diagram demonstrating study inclusion criteria. Abbreviations: RT, radiation therapy; OSH, outside hospital; HNC, head and neck cancer; CVE, cerebrovascular event; US, ultrasound; ENT, otolaryngology; CAS, carotid artery stenosis. 

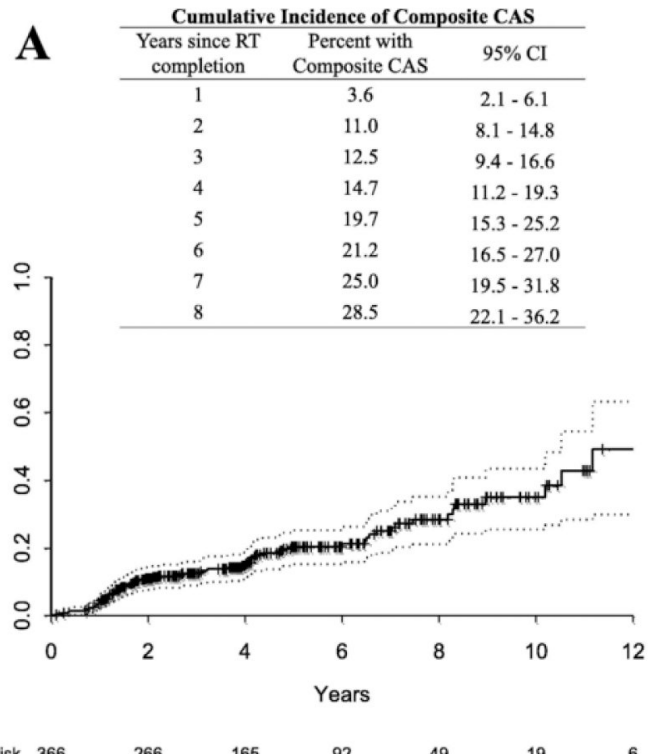

Fig. 2.

(A) Cumulative incidence of composite carotid artery stenosis (asymptomatic CAS, stroke, or TIA) is shown with $95 \%$ CI provided for years post-RT completion with number at risk 20. (B) Cumulative incidence of asymptomatic carotid artery stenosis is shown with $95 \%$ CI provided for years post-RT completion with number at risk $\geq 20$. 

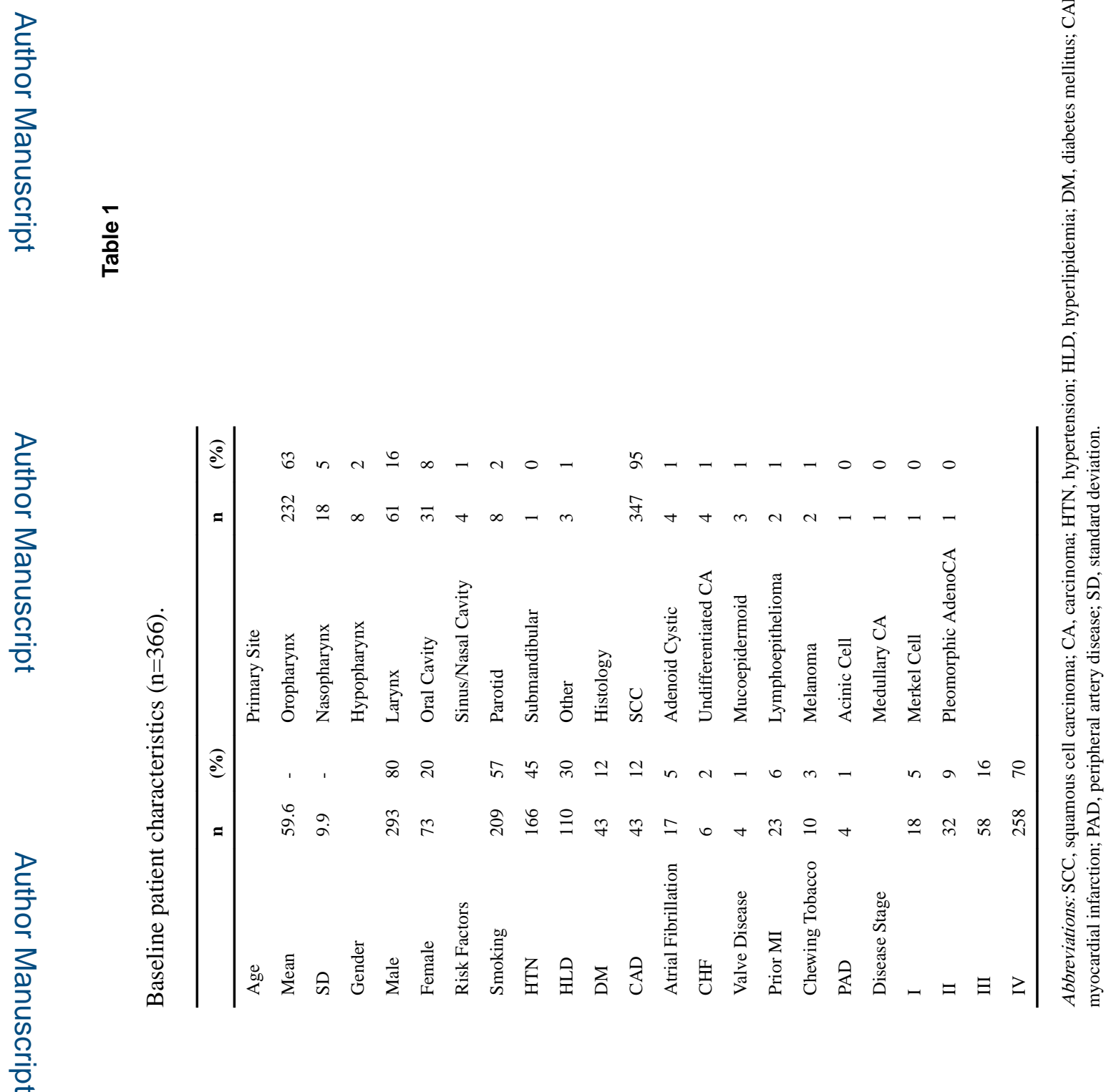
Oral Oncol. Author manuscript; available in PMC 2019 November 04. 


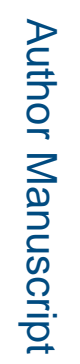




\section{Table 3}

Analysis for composite carotid artery stenosis per patient.

\begin{tabular}{lcll}
\hline Factor (reference value) & HR & 95\% CI & p-value \\
\hline (A) Univariate analysis of composite CAS & & \\
Age (years, continuous) & 1.03 & $1.003-1.05$ & $0.03^{*}$ \\
Sex (F) & 0.5 & $0.2-1.04$ & 0.06 \\
Smoking (No) & 1.7 & $1.1-2.8$ & $0.03^{*}$ \\
HTN (No) & 1.3 & $0.8-2.1$ & 0.23 \\
HLD (No) & 1.6 & $0.98-2.5$ & 0.06 \\
DM (No) & 2.4 & $1.4-4.2$ & $0.002^{*}$ \\
CAD (No) & 2.3 & $1.3-4.1$ & $0.007^{*}$ \\
Heart disease (No) & 1.02 & $0.3-2.8$ & 0.97 \\
PAD (No) & 2.8 & $0.7-11.3$ & 0.16 \\
Neck dissection (No) & 0.99 & $0.6-1.6$ & 0.96 \\
Chemotherapy (No) & 1.02 & $0.6-1.8$ & 0.95 \\
RT Side (Bilateral) & 1.4 & $0.7-2.8$ & 0.28 \\
Total RT Dose (Gy, continuous) & 0.98 & $0.95-1.02$ & 0.36 \\
Factor (reference value) & HR & $95 \%$ CI & p-value \\
(B) Multivariate analysis of composite CAS & \\
Age (years, continuous) & 1.0 & $0.99-1.05$ & 0.18 \\
Sex (F) & 0.5 & $0.3-1.1$ & 0.10 \\
Smoking (No) & 1.5 & $0.9-2.5$ & 0.12 \\
HLD (No) & 1.2 & $0.7-2.1$ & 0.42 \\
DM (No) & 1.9 & $1.1-3.4$ & $0.03^{*}$ \\
CAD (No) & 1.4 & $0.8-2.8$ & 0.27 \\
\hline
\end{tabular}

Abbreviations: CAS, carotid artery stenosis; HR, hazard ratio; CI, confidence interval; F, female; RT, radiation therapy 


\section{Table 4}

Univariate analysis for composite carotid artery stenosis per carotid artery.

\begin{tabular}{llll}
\hline Factor (Reference) & HR & $\mathbf{9 5 \%}$ CI & p-value \\
\hline Age (years, continuous) & 1.02 & $0.999-1.04$ & 0.07 \\
Sex (F) & 0.5 & $0.3-1.04$ & 0.07 \\
Smoking (No) & 1.7 & $1.1-2.7$ & $0.03^{*}$ \\
HTN (No) & 1.3 & $0.8-2.0$ & 0.3 \\
HLD (No) & 1.6 & $1.03-2.6$ & $0.04^{*}$ \\
DM (No) & 2.8 & $1.6-4.8$ & $0.0003^{*}$ \\
CAD (No) & 2.4 & $1.4-4.2$ & $0.002^{*}$ \\
Heart Disease (No) & 0.9 & $0.3-2.5$ & 0.86 \\
PAD (No) & 3.6 & $1.1-11.6$ & $0.03^{*}$ \\
Neck dissection (No) & 1.4 & $0.9-2.4$ & 0.17 \\
Chemotherapy (No) & 0.9 & $0.5-1.6$ & 0.73 \\
RT Side: Contralateral & 1.9 & $0.7-5.3$ & 0.21 \\
Entire carotid: & & & \\
Max dose per 10Gy & 1.001 & $0.999-1.002$ & 0.41 \\
Mean dose per 10Gy & 1.00 & $0.999-1.002$ & 0.56 \\
V40 & 1.003 & $0.995-1.01$ & 0.54 \\
V50 & 1.002 & $0.9-1.1$ & 0.96 \\
V60 & 1.003 & $0.994-1.01$ & 0.54 \\
V70 & 1.04 & $0.9-1.2$ & 0.45 \\
Carotid bulb: & & & \\
Max dose per 10Gy & 1.001 & $0.999-1.002$ & 0.36 \\
Mean dose per 10Gy & 1.00 & $0.999-1.002$ & 0.49 \\
V40 & 1.003 & $0.995-1.01$ & 0.50 \\
V50 & 1.006 & $0.9-1.1$ & 0.85 \\
V60 & 1.002 & $0.996-1.01$ & 0.46 \\
V70 & 1.03 & $0.97-1.1$ & 0.39 \\
\hline & & & \\
\hline
\end{tabular}

